\title{
Tradução, adaptação transcultural para a língua portuguesa e validação do instrumento Undergraduate Clinical Education Environment Measure (Uceem)
}

\author{
Translation, cross-cultural adaptation to the Portuguese language and validation of the Undergraduate Clinical \\ Education Environment Measure Instrument (Uceem)
}

\author{
Milena da Costa ${ }^{1}$ (D) $\mid$ mi.enfermagem.fpp@gmail.com \\ Christian Boller ${ }^{1}$ (D) christian.boller@fpp.edu.br \\ Ivete Palmira Sanson Zagonel' (1) ivete.zagonel@fpp.edu.br
}

\begin{abstract}
RESUMO
Introdução: O ambiente/clima educacional para o ensino clínico destinado a estudantes de Medicina e Enfermagem influencia a percepção deles sobre os aspectos significativos de satisfação no ambiente clínico de aprendizagem.

Objetivo: Este estudo teve como objetivos adaptar transculturalmente com validade de face, conteúdo e constructo o instrumento Undergraduate Clinical Education Environment Measure (UCEEM) para o português do Brasil e validar a versão traduzida e adaptada com estudantes de Enfermagem e Medicina que vivenciam o ensino clínico durante a formação.

Método: Trata-se de um estudo metodológico para tradução, adaptação e validação transcultural, processo dividido em seis fases propostas por Beaton. As etapas consistiram em tradução, síntese das traduções, retrotradução, revisão pelo comitê de experts, pré-teste e envio da versão final do instrumento adaptado à autora do instrumento. Participaram do processo cinco tradutores bilíngues (três para tradução e dois para retrotradução) e oito profissionais experts que atuam como docentes e possuem título de mestre e/ou doutor. A validação foi realizada em dois momentos. No primeiro, realizaram-se as equivalências semântica, idiomática, cultural e conceitual (pré-teste) com 30 estudantes de Enfermagem e Medicina vivenciando o ensino clínico. No segundo, efetuou-se a validação com avaliação das propriedades psicométricas de 161 estudantes desses mesmos cursos.

Resultado: A adaptação transcultural foi realizada por oito profissionais experts da área, além contar com a análise e as considerações da pesquisadora e autora do instrumento original. O resultado dessa etapa conduziu à versão final do instrumento em português, que apresenta 26 itens divididos em duas dimensões e quatro subescalas, as quais foram mantidas conforme original. Após o preenchimento do instrumento por estudantes de Medicina e Enfermagem, a análise de Teoria de Resposta ao Item revelou confiabilidade, determinada pelo alfa de Cronbach, de 0,917. No teste de correlação de Spearman, os resultados apontaram a existência de correlação positiva entre as questões.
\end{abstract}

Conclusão: A versão em português do instrumento UCEEM mostrou-se adequada e útil para avaliar a satisfação com o ambiente educacional no ensino clínico pela percepção dos estudantes de Medicina e Enfermagem.

Palavras-chave: Estudos de Validação como Assunto; Ensino; Educação Médica; Educação em Enfermagem.

\section{ABSTRACT}

Introduction: The educational environment for the clinical teaching of undergraduate medical and nursing students influences the students' perception of significant aspects of satisfaction in the clinical learning environment.

Objective: To transculturally adapt, with the concept, content and construct validity, the instrument "Undergraduate Clinical Education Environment Measure - UCEEM" into the Brazilian Portuguese language and validate the translated and adapted version; involving medical and nursing students, while experiencing clinical teaching during their undergraduate course.

Method: This is a methodology study aimed at the translation, adaptation and transcultural validation process, which is divided into six steps, as proposed by Beaton. The steps consisted in the translation, translation synthesis, back-translation, proofreading by the experts committee, pre-testing and submission of the final version of the instrument to the author. Five bilingual translators participated in this process (three for the translation and two for the back-translation), as well as eight expert professionals who work as academic faculty and have Master's and Doctoral degrees. The validation was carried out at two moments: the first one by using semantic, idiomatic, cultural and conceptual equivalence (pre-test), involving 30 medical and nursing students going through clinical teaching; and the second moment, the validation and assessment of the psychometric properties with 161 students from these same courses.

Result: The transcultural adaptation carried out by eight expert professionals in this area was performed based on the experts' considerations, in addition to the analysis and considerations of the researcher and author of the original instrument. The result of this phase led to the final version of this instrument in Brazilian Portuguese, which shows 26 items divided into two dimensions and four subscales that were maintained according to its original form. Following the filling out of the instrument by the nursing and medical students, the analysis of the Theory of Response revealed to be reliable, as determined by a Cronbach's alpha of 0.917. According to Spearman's correlation test, the results indicate a positive correlation between the questions.

Conclusions: The Portuguese version of the UCEEM instrument showed to be adequate and useful to evaluate the satisfaction with the educational environment in clinical teaching according to the perception of medical and nursing students.

Keywords: Validation Studies as Topic; Teaching; Education, Medical; Education, Nursing.

1 Faculdades Pequeno Príncipe, Curitiba, Paraná, Brasil.

Editora-chefe: Rosiane Viana Zuza Diniz. $\quad$ Editor associado: Pedro Tadao Hamamoto Filho.

Recebido em 08/06/21; Aceito em 28/10/21. Avaliado pelo processo de double blind review. 


\section{INTRODUÇÃO}

O ambiente educacional (AE) oportuniza aos estudantes aprender com inter-relação entre os colegas, professores e membros da equipe de saúde, bem como estimula a autoconfiança. As percepções dos estudantes sobre a qualidade do $\mathrm{AE}$ revelam informações que estão diretamente relacionadas ao processo de ensino-aprendizagem'.

O conceito de AE está ligado a todo e qualquer contexto em que ocorrem o ensino e o aprendizado. Sinônimos que também são utilizados para AE incluem "atmosfera educacional" e"clima educacional". O AE tem impacto e influência no processo ensino-aprendizagem, bem como na qualidade do trabalho a ser desenvolvido pelos graduandos e egressos ${ }^{2,3}$. O conceito de "clima educacional" traduz a forma como o estudante percebe o ambiente, ou seja, informa sobre o seu comportamento ante os diferentes desafios das atividades educacionais. Um mesmo ambiente pode ser amigável para um estudante e/ou ameaçador para outro².

Diversos fatores influenciam no processo ensinoaprendizagem, como as instalações, a estrutura física e material, o relacionamento interpessoal, a metodologia de ensino, as dimensões ética e social, os serviços acadêmicos, os programas de assistência social, a política de avaliação institucional, a qualificação docente, a qualificação técnico-administrativa, a relação professor/estudante, entre outros. Essas influências integram elementos constitutivos que favorecem a formação de profissional crítico e reflexivo, e reconhecem o estudante como sujeito ativo ${ }^{3,4}$.

É necessário estimular o estudante a criar um pensamento reflexivo, sensível às problemáticas atuais, baseado e fundamentado em sua vida na sociedade em que está inserido, em sua própria prática social, relacionando a teoria com a prática 5 . O professor, ciente de seu papel no $A E$, adapta o planejamento das atividades acadêmicas às condições dos estudantes, busca aperfeiçoar o seu trabalho docente e mediar a construção do conhecimento por parte dos discentes ${ }^{6}$.

O clima educacional, na percepção dos estudantes, tem impacto na qualidade de aprendizagem. A readequação do ambiente pode ser uma estratégia eficaz para atingir os objetivos organizacionais, pois contempla tanto as necessidades da instituição de ensino como as expectativas e ideias pessoais do estudante, eliminando os efeitos negativos de insatisfação com o ambiente formativo e o curso ${ }^{4}$. Ao docente, cabe atualizar seus conhecimentos, se preparar e estar seguro em transmitir suas experiências, com o intuito de orientar o acadêmico em sua performance, na qualidade de suas ações, articulando teoria e prática, como processo dinâmico, interativo. $\mathrm{O}$ ensino clínico tem como objetivo desenvolver competências, viabilizando uma aprendizagem significativa inter-relacionada com o AE de ensino ${ }^{7-9}$.
A aprendizagem em cenários clínicos e com inserção precoce pode direcionar os currículos médicos para o contexto social da prática, ajudar na transição dos acadêmicos para o ambiente clínico, motivá-los, torná-los mais confiantes para lidar com os pacientes e aumentar a autoconsciência e consciência dos outros ${ }^{10}$.

Em pesquisa para comparar a percepção de suporte social e a avaliação do AE entre dois grupos de estudantes de Medicina, sendo um grupo com apoio de mentoria, utilizaramse a Escala de Satisfação com o Suporte Social (ESSS) para a avaliação da percepção do suporte social e a Dundee Ready Education Environment Measure (DREEM) para a avaliação do AE. Observou-se um perfil mais crítico em relação à percepção de suporte social e ao ambiente acadêmico entre os estudantes. A mentoria pode auxiliar de forma positiva o percurso dos estudantes, seja tornando-os mais críticos em relação ao ensino e à sua forma de aprender, seja fornecendo ferramentas de apoio acadêmico ${ }^{11}$.

Quando se explorou a literatura, percebeu-se que existem instrumentos desenvolvidos para avaliar o ambiente de aprendizagem, a maioria é internacional e poucos são direcionados a avaliar esse ambiente na percepção dos estudantes em ambiente de aprendizagem clínica.

Os Ambientes de Aprendizagem Clínica (Clinical Learning Environments - CLE) abrangem uma diversidade de elementos, como oportunidades de aprendizagem, definições de papéis e atitudes em relação ao ensino e aos pacientes, e todos podem influenciar a aprendizagem, o bem-estar e a satisfação do estudante. As percepções dos acadêmicos sobre o CLE também afetam a capacidade de atingir as habilidades necessárias para progredir no treinamento e a forma como eles praticam após terminar o treinamento ${ }^{12}$.

Entre os instrumentos psicométricos disponíveis, temse o Undergraduate Clinical Education Environment Measure (UCEEM) criado por Strand et al. $^{13}$. O instrumento avalia a percepção de estudantes sobre os aspectos significativos do clima de aprendizagem no ambiente de ensino clínico, além de fornecer um feedback, para os locais de atuação e as instituições educacionais, sobre como os estudantes percebem as dimensões sociais, emocionais e cognitivas do ambiente de aprendizado $^{13}$. A UCEEM pode ser usado como uma medida de linha de base e posteriormente para acompanhamento e avaliação da eficácia de intervenções para melhorar o ambiente de aprendizagem clínica ${ }^{12}$.

Diante disso e devido à ausência de instrumentos validadosqueabordemotema emquestãonalíngua portuguesa, este estudo teve como objetivos adaptar transculturalmente com validade de face, conteúdo e constructo o instrumento UCEEM para o português do Brasil e validar a versão traduzida 
e adaptada com estudantes de Enfermagem e Medicina que vivenciam o ensino clínico durante a formação.

\section{MÉTODO}

Trata-se de um estudo metodológico que se refere aos métodos de obtenção, organização e análise dos dados. Por meio da adaptação transcultural, da elaboração, da validação e da avaliação do instrumento, os resultados obtidos revelam as diferenças ou similaridades entre as culturas envolvidas ${ }^{14,15}$. A autora do instrumento autorizou a tradução, a adaptação e a validação dele e seu uso no Brasil.

\section{Sobre o instrumento}

$O$ instrumento UCEEM foi criado para apreender a percepção de satisfação dos estudantes do AE durante a realização do ensino clínico. Esse instrumento pode ser usado para reconhecer as qualidades do ambiente de aprendizagem ou identificar as ações que devem ser tomadas, favorecendo o aprendizado por meio da oferta de um ambiente de qualidade, oportunidades de crescimento acadêmico, estrutura e equipamentos adequados. O UCCEM permite avaliar, segundo a percepção dos estudantes, como eles se sentem no ambiente de ensino clínico, como são recebidos pela equipe e se a estrutura coloca os conhecimentos teóricos na prática ${ }^{13}$. Apresenta 26 itens divididos em duas dimensões e quatro subescalas ou fatores:

- Dimensão 1: "Experiência da aprendizagem", com F1 "oportunidades de aprender na e pela prática e qualidade da supervisão" e F2 "preparação para a entrada do estudante".

- Dimensão 2: "Participação social", com F3 "padrões de interação no cenário de prática" e F4"tratamento igualitário".

O F1 refere-se às percepções dos estudantes sobre as oportunidades de aprender na e pela experiência prática e sobre o processo de construção de significado dessas experiências, com supervisores e pares (questões 3, 4, 5, 6, 13, $14,15,16,17,18,26)$. O F2 reflete as percepções dos discentes sobre a preparação do cenário de prática $(A E)$ para a entrada dos deles e como os supervisores/preceptores devem tornar suas experiências pessoais de aprendizagem baseadas em práticas significativas e relevantes para o currículo (questões $1,2,9,10,11,12)$. O F3 e F4 contêm variáveis que mapeiam vários aspectos relacionados à participação social e equidade (questões do F3: 7, 8, 19, 20, 21, 22; questões de F4: 23, 24 e 25). Para a utilização no Brasil, o instrumento passou pelas etapas indicadas na Figura 1.

\section{Etapa de tradução e adaptação transcultural}

Para a tradução e adaptação transcultural do instrumento UCEEM, seguiram-se os passos metodológicos de tradução, síntese das traduções, retrotradução, revisão por experts, préteste e validação ${ }^{16}$.

A tradução para o português foi realizada por três tradutores bilíngues que tinham conhecimento dos objetivos propostos da pesquisa e dos conceitos envolvidos. $\mathrm{Na}$ retrotradução, participaram dois tradutores bilíngues que não tinham conhecimento dos objetivos e conceitos envolvidos. A avaliação das equivalências semântica, idiomática, cultural e conceitual dos itens do UCEEM foi realizada por um comitê de oito profissionais experts, sendo cinco médicos, dois enfermeiros e um biomédico, os quais atuam como docentes e possuem título de mestre e/ou doutor. Enviou-se via e-mail o Termo de Consentimento Livre e Esclarecido (TCLE) para que os experts pudessem assiná-lo. Enviaram-se ainda uma planilha em Excel com os itens da versão original do instrumento, o consenso dos tradutores e pesquisadores (etapas 1 e 2), o consenso dos retrotradutores e pesquisadores (etapa 3), com uma coluna em branco para que os experts pudessem descrever as considerações necessárias sobre cada item do instrumento, e

Figura 1. Representação das etapas de tradução, adaptação transcultural e validação do UCEEM.

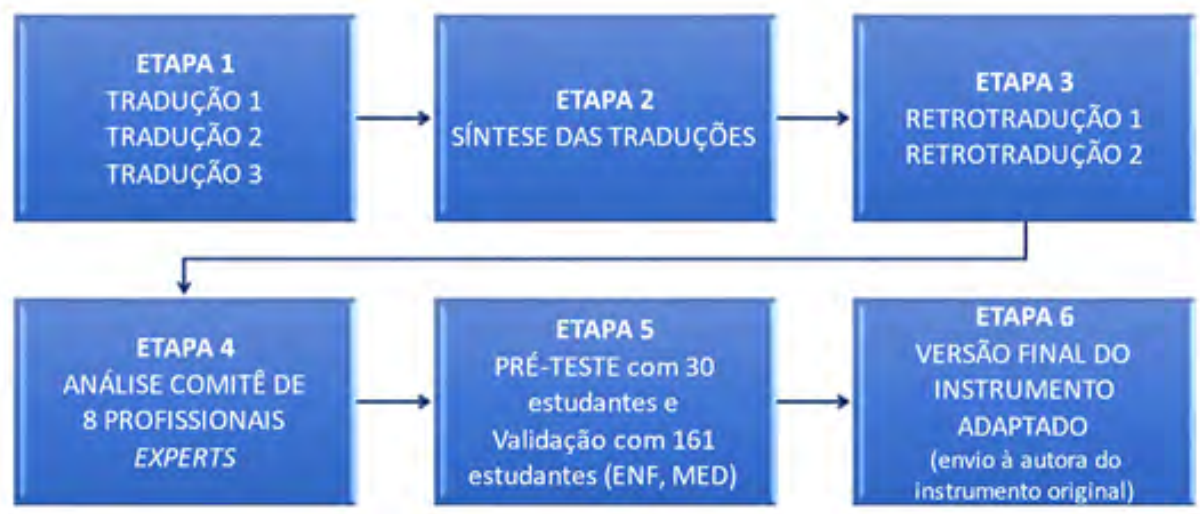

Fonte: Adaptada de Beaton et al. ${ }^{16}$. 
o consenso com a opinião deles e as alterações sugeridas, para melhor compreensão do público-alvo.

Os resultados das etapas 1, 2 e 3 demonstrados no Quadro 1 apresentam a versão original, as traduções (T1, T2 e T3) e o consenso até a questão 1 , bem como as retrotraduções (R1 e R2) da versão original do instrumento UCEEM, a título de ilustração.

As etapas de tradução, retrotradução e avaliação de experts (etapa 4) foram analisadas pela pesquisadora, considerando as sugestões de cada especialista. Realizaram-se feedback e troca de mensagens com os tradutores envolvidos até a obtenção do consenso. Após a retrotradução realizada pelos dois tradutores, cada um enviou por e-mail suas respectivas traduções; a pesquisadora agrupou a versão préfinal do instrumento em português e as duas retrotraduções em planilha do Excel; foi reenviada aos tradutores a planilha para que analisassem a outra retrotradução e descrevessem suas considerações, analisando cada item; escolhiam qual seria a melhor tradução e quais as considerações, adequações que achavam necessárias fazer, com o intuito de deixar o instrumento mais parecido com o original, sem alterar o sentido de cada frase.

As considerações do profissional expert 2 (EX2) referiramse ao item 1:
[...] a retrotradução não traduz o termo inicial utilizado, nem passa a mesma mensagem. Reinforcement talvez não seja o termo mais apropriado. O termo "reforço" escolhido pelos pesquisadores talvez devesse ser trocado por "orientações iniciais". O item 1 ficando da seguinte forma: "I recieved a useful information/ introduction to this placement/". Eu recebi orientações iniciais adequadas".

Por sua vez, o EX8 também questionou o item 1, mas com relação à palavra placement, que, para ele, descreve que "refere-se ao estágio".

\section{Etapas de validação}

A validação (etapa 5) foi realizada em dois momentos. No primeiro, realizaram-se as equivalências semântica, idiomática, cultural e conceitual (pré-teste) com 30 estudantes, sendo 15 de Enfermagem e 15 de Medicina, vivenciando o ensino clínico, do segundo ao décimo segundo períodos, nos meses de setembro e outubro 2019. O segundo momento consistiu na validação com 161 estudantes dos mesmos cursos, nos meses de dezembro de 2019 a fevereiro de 2020. A coleta de dados para validação foi desenvolvida com estudantes de instituição de ensino superior (IES) filantrópica localizada em Curitiba, no Paraná. O estudo foi aprovado pelo Comitê de Ética em Pesquisa com Seres Humanos, sob Parecer n 2.866 .282 e

Quadro 1. S Segmento das traduções (T1, T2 e T3) e retrotraduções (R1 e R2) da versão original do instrumento UCEEM.

\begin{tabular}{|c|c|c|c|c|c|}
\hline Versão original & $\mathrm{T} 1$ & $\mathrm{~T} 2$ & T3 & Pesquisadora & $\begin{array}{c}\text { Consenso } \\
\mathrm{T} 1, \mathrm{~T} 2, \mathrm{~T} 3 \text { e pesquisadora }\end{array}$ \\
\hline $\begin{array}{l}\text { Título: } \\
\text { Undergraduate } \\
\text { Clinical Education } \\
\text { Environment } \\
\text { Measure }\end{array}$ & $\begin{array}{l}\text { Título: Medida } \\
\text { do Ambiente de } \\
\text { Educação Clínica } \\
\text { de Graduação }\end{array}$ & $\begin{array}{l}\text { Título: Medição } \\
\text { Ambiental na } \\
\text { Graduação em } \\
\text { Educação Clínica }\end{array}$ & $\begin{array}{l}\text { Título: Medida } \\
\text { do Ambiente de } \\
\text { Educação Clínica } \\
\text { de Graduação }\end{array}$ & $\begin{array}{l}\text { Título: Medida } \\
\text { do Ambiente de } \\
\text { Ensino Clínico de } \\
\text { Graduação }\end{array}$ & $\begin{array}{l}\text { Título: Medida do } \\
\text { Ambiente de Ensino } \\
\text { Clínico na Graduação }\end{array}$ \\
\hline $\begin{array}{l}\text { 1. I received useful } \\
\text { induction to this } \\
\text { placement. }\end{array}$ & $\begin{array}{l}\text { 1. Eu recebi } \\
\text { indução útil para } \\
\text { esta colocação. }\end{array}$ & $\begin{array}{l}\text { 1. Recebi indução } \\
\text { útil para este } \\
\text { posicionamento. }\end{array}$ & $\begin{array}{l}\text { 1. Recebi indução } \\
\text { útil para esta } \\
\text { colocação. }\end{array}$ & $\begin{array}{l}\text { 1. Recebi reforço } \\
\text { útil para esta } \\
\text { colocação. }\end{array}$ & $\begin{array}{l}\text { 1. Recebi reforço útil } \\
\text { para esta colocação. }\end{array}$ \\
\hline \multicolumn{6}{|c|}{$\begin{array}{l}\text { Versão pré-final do instrumento UCEEM T1, T2, T3 e da pesquisadora, retrotradução (R1 e R2) e consenso dos retrotradutores e da } \\
\text { pesquisadora. }\end{array}$} \\
\hline $\begin{array}{c}\text { Versão pré-final } \\
\text { T1, T2, T3 e } \\
\text { pesquisadora }\end{array}$ & R1 & $\mathrm{R} 2$ & Pesquisadora & $\begin{array}{l}\text { Consenso R1, R2 e } \\
\text { pesquisadora }\end{array}$ & \\
\hline $\begin{array}{l}1 \text { Recebi reforço útil } \\
\text { para esta colocação. }\end{array}$ & $\begin{array}{l}1 . \text { I received } \\
\text { useful } \\
\text { reinforcement in } \\
\text { this placement. }\end{array}$ & $\begin{array}{l}\text { 1. I received helpful } \\
\text { reinforcement for } \\
\text { this placement. }\end{array}$ & & $\begin{array}{l}1 . \text { I received } \\
\text { useful } \\
\text { reinforcement in } \\
\text { this placement. }\end{array}$ & \\
\hline
\end{tabular}

Fonte: Elaborado pelos autores. 
Certificado de Apresentação para Apreciação Ética (CAAE) nº 96760118.2.0000.5580.

No pré-teste, o instrumento foi aplicado de forma presencial aos estudantes, com o intuito de acompanhar o momento do preenchimento do instrumento. Entregou-se o TCLE para leitura e assinatura, indicando o aceite em participar do estudo, a todos os estudantes nos períodos e nas disciplinas selecionados. Escolheu-se uma disciplina/unidade curricular de cada período dos cursos de Enfermagem e Medicina, com autorização do coordenador do curso e docente responsável. Somente assinavam o TCLE aqueles estudantes, elegíveis aos critérios de inclusão, que estivessem de acordo com a realização da pesquisa. O tempo médio para preenchimento do instrumento foi de aproximadamente 20 minutos. Os estudantes descreveram no próprio instrumento as dificuldades ou sugestões com relação à clareza.

$\mathrm{Na}$ validação (etapa 5), o instrumento foi aplicado via on-line, com retorno de 161 estudantes, pela ferramenta do Google Forms. Os estudantes que aceitaram participar da pesquisa consentiram pelo TCLE disponibilizado, sendo após direcionados para o instrumento.

A pesquisadora nessa etapa, para obter a amostra, após liberação dos coordenadores dos cursos, passou em todas as turmas dos cursos de Enfermagem e Medicina elegíveis aos critérios de inclusão, durante dois dias, para explicar a pesquisa, os objetivos, o instrumento que estava sendo validado e a importância da participação. Eis os critérios de inclusão: estar matriculado no curso de Enfermagem ou Medicina, do segundo ao $12^{\circ}$ período, estar vivenciando o ensino clínico e ser maior de 18 anos. Excluíram-se os estudantes que não estavam presentes no dia da aplicação do instrumento (fase presencial) e aqueles com matrícula trancada ou matriculados no primeiro período.

\section{Análise dos dados do instrumento validado pelos especialistas}

O instrumento já validado pelos especialistas (tradução e retrotradução, conforme Quadro 1) foi aplicado aos estudantes em duas etapas com o objetivo de testá-lo na prática. Analisaramse os resultados por meio da Análise Fatorial Confirmatória (Confirmatory Factor Analysis - CFA), com o uso do programa IBM SPSS AMOS 26.0.0, e também pela Teoria de Resposta ao Item (TRI), por meio do complemento elRT (versão 1.2.0; http:// libirt.sf.net) para o software Microsoft Excel (Microsoft 365), com análise pelo Modelo de Resposta Gradual de Samejima ${ }^{17}$. Essa análise permite a obtenção do índice de confiabilidade do instrumento, determinado pelo alfa de Cronbach, bem como do índice de discriminação, o qual verifica a relação entre os escores globais de um respondente e a resposta em cada item individual ${ }^{18}$. A escala Likert é baseada em perguntas que foram transformadas em números para a análise. Após o cálculo, cada pergunta é transformada em uma escala que indica o valor de concordância dos avaliadores.

O modelo de Samejima permite obter a Curva Característica Operacional (Operating Characteristic Curve - OCC) de cada item. De forma qualitativa, o formato cônico da curva permite prever o poder avaliativo da questão, indicando se ela é ou não uma boa questão para avaliar o que se propõe. $\mathrm{O}$ somatório das curvas de cada item indica se o instrumento em si está adequado para avaliar o traço latente proposto, que consiste na satisfação dos estudantes com o AE de ensino clínico.

\section{RESULTADOS}

As análises semântica, idiomática, cultural e conceitual foram o foco da validação realizada pelos experts. Essas análises envolveram respostas qualitativas dadas por cada especialista. Eis alguns exemplos desse tipo de contribuição: 1. “O ideal seria ocultar o sujeito 'eu' no instrumento", 2. "Na verdade, a palavra original useful poderia ser substituída por helpful", 3. "A palavra 'encorajado' poderia ser empregada em substituição à palavra 'incentivado', adequando-se ao sentido do enunciado".

A partir dessas contribuições, os autores analisaram todas as respostas e optaram consensualmente pelas respostas prevalentes; assim, o questionário foi adquirindo a característica da versão final. Pelo seu caráter qualitativo, não foram realizadas análises estatísticas, visto que dependem de valores numéricos de concordância.

A análise dos respondentes ao UCEEM revela consistência interna do instrumento traduzido para o português brasileiro. Nas duas etapas de aplicação do UCEEM, tanto no pré-teste quanto na validação, os estudantes relataram que o instrumento se apresentava claro, utilizando linguagem simples, com perguntas objetivas, de fácil compreensão e autoexplicativas, diretamente relacionadas ao ambiente de ensino clínico que eles vivenciavam, não havendo, portanto, necessidade de alterá-lo.

Para a CFA, foi adotado o modelo de quatro fatores originalmente proposto pela autora, cujo resultado apresentou bom ajuste ao modelo. Os valores dos índices de ajuste para o questionário estão na Tabela 1, bem como na representação gráfica da CFA na Figura 2. Os índices possuem valores que variam de 0 a 1, e quanto menor for o índice Root Mean Square Error of Approximation (RMSEA), melhor será o ajuste do modelo. Já os outros índices apresentados indicam que valores maiores demonstram melhor ajuste do modelo.

A análise de TRI, realizada individualmente para cada fator ( $n=161)$, apresentou os seguintes valores: $F 1=0,858$, $\mathrm{F} 2=0,827, \mathrm{~F} 3=0,816$ e $\mathrm{F} 4=0,882$. A autora do instrumento original propôs um modelo multidimensional para Medida do Ambiente de Ensino Clínico na Graduação. Os valores apontam 
Tabela 1. Índice de ajuste ao modelo proposto pela CFA

\begin{tabular}{cc}
\hline Índice & Valor \\
\hline RMSEA & 0,088 \\
NFI & 0,704 \\
IFI & 0,811 \\
TLI & 0,766 \\
CFI & 0,805 \\
\hline
\end{tabular}

RMSEA: Root Mean Square Error of Approximation (Raiz da Média dos Quadrados dos Erros de Aproximação); NFI: Bentler and Bonnet's Normed Fit Index (Índice de Ajuste Normalizado de Bentler e Bonnet); IFI - Bollen's Incremental Fit Index (Índice de Ajuste Corrigido de Bollen); TLI: Tucker-Lewis Index (Índice de Tucker e Lewis); CFI: Bentler's Comparative Fit Index (Índice de Ajuste Comparativo de Bentler). Fonte: Elaborada pelos autores.

Gráfico 1.Curva total dos itens obtidos dos participantes da pesquisa.

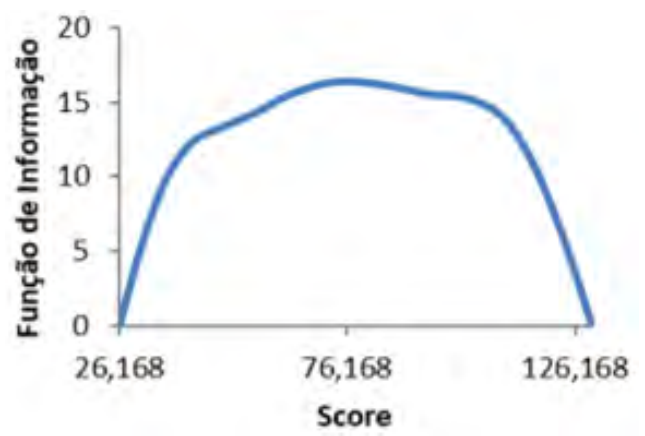

Fonte: Dados da pesquisa.

que o modelo ora traduzido também pode ser considerado multidimensional com quatro fatores, conforme proposto pelo instrumento original. Na análise de correlação de Spearman, os dados demonstraram que existe correlação positiva entre as questões $(p<0,05)$, o que contribuiu para o alto valor de alfa calculado para cada fator do instrumento.

A análise da curva total do instrumento permitiu observar que os estudantes, embora desenvolvam atividades acadêmicas no mesmo ambiente escolar, a percepção deles variou de muito baixa até muito alta. Isso se refletiu nos diferentes níveis de habilidades observados no instrumento aplicado. Para cada ponto de escore (traço latente que variou no instrumento de 55 até 126), tem-se uma função da quantidade de informação que pode ser obtida pelo instrumento. Assim, observa-se que, em todos os possíveis escores que o instrumento pode apresentar, os valores são elevados. Essa interpretação é demonstrada no Gráfico 1 que apresenta o resultado da curva total dos itens.

A partir dos escores individuais de cada participante, foi possível estabelecer que o traço latente observado (satisfação com o ambiente de ensino clínico) pode ser observado e analisado com esse instrumento. A graduação dos valores
Figura 2. Análise Fatorial Confirmatória (CFA) para a versão brasileira do instrumento UCEEM.

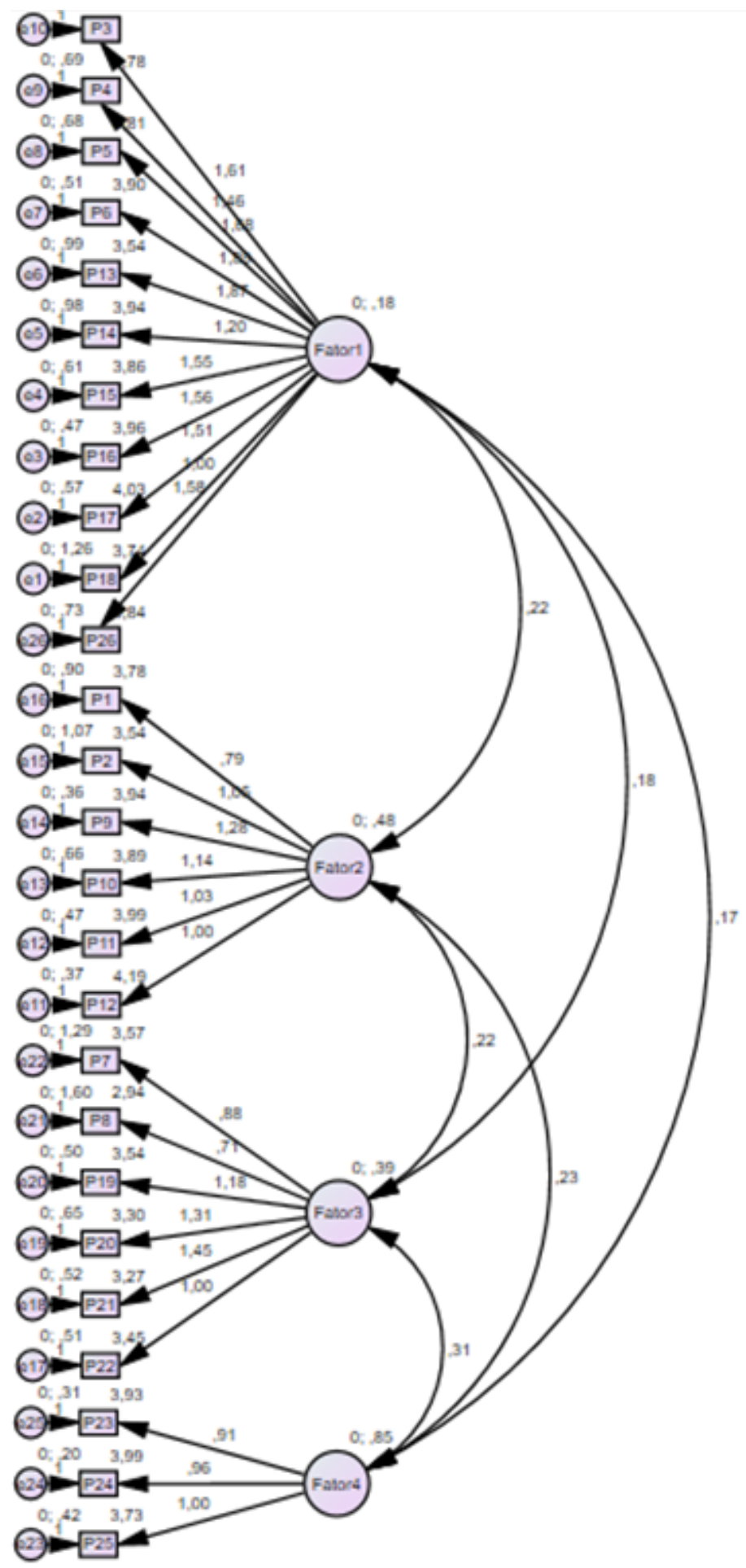

Fonte: Elaborada pelos autores.

mínimos e máximos analisados foi de $-2,290$ até 2,601, o que equivale ao escore do instrumento de 55 até 126 . Considerando que, se um estudante respondesse $5 \mathrm{em}$ todas as alternativas, $\mathrm{o}$ escore seria de 130. É possível afirmar que o instrumento avalia a satisfação com o AE de ensino clínico de forma positiva. O escore gerado pelo instrumento estima de forma confiável uma multiplicidade de resultados. No lado oposto, caso o participante 
respondesse 1 em todas as alternativas, o valor seria de exatos 26 pontos, equivalente ao número de questões. Assim, também é possível argumentar que o instrumento permite avaliar os estudantes insatisfeitos com o AE de ensino clínico.

Outro item analisado é o índice de discriminação, valor que varia de 0 a 1 e indica o nível de clareza de cada item. $O$ Quadro 2 apresenta os valores médios de cada questão e seus respectivos desvios padrão, ordenados em ordem crescente pelo seu índice de discriminação.

Esse índice evidencia o nível de certeza que o participante tem em diferenciar um item de outro. Quanto maior for o valor, melhor será o nível de discriminação. Assim, observa-se que a questão 16 é a que melhor permite distinguir o participante quanto a discordar totalmente (1) e concordar plenamente (5).

\section{DISCUSSÃO}

A validação do instrumento UCEEM denominado, após a tradução, Medida do Ambiente de Ensino Clínico na Graduação (Anexo 1) possibilitou quantificar e analisar características subjetivas dos envolvidos na pesquisa. O uso de instrumentos validados na área da saúde permite verificar a necessidade de intervenção em algum processo ou método. A validação de

Quadro 2. Valores de média, desvio e índice de discriminação por questão, em ordem crescente de índice de discriminação.

\begin{tabular}{|c|c|c|c|}
\hline Item & Média & Desvio & Discriminação \\
\hline $\begin{array}{l}\text { 18. Aqui, tenho a oportunidade de aprender junto com outros estudantes da } \\
\text { área de saúde. }\end{array}$ & 3,744 & 1,200 & 0,337 \\
\hline $\begin{array}{l}\text { 8. Há espaço físico suficiente para o número de estudantes da área de saúde } \\
\text { alocados aqui. }\end{array}$ & 2,944 & 1,338 & 0,354 \\
\hline $\begin{array}{l}\text { 23. Aqui, todos são tratados com igual respeito e dignidade, independentemente } \\
\text { de sua origem cultural. }\end{array}$ & 3,925 & 1,007 & 0,437 \\
\hline $\begin{array}{l}\text { 14. Sinto-me à vontade em tirar dúvidas com meus supervisores de qualquer } \\
\text { questão que deseje. }\end{array}$ & 3,944 & 1,108 & 0,447 \\
\hline $\begin{array}{l}\text { 25. Aqui, todos são tratados com igual respeito e dignidade, independentemente } \\
\text { de sua profissão. }\end{array}$ & 3,731 & 1,128 & 0,454 \\
\hline 7. Eu tenho acesso adequado aos computadores. & 3,569 & 1,258 & 0,455 \\
\hline 1. Recebi instruções iniciais úteis para este local de atuação. & 3,781 & 1,099 & 0,465 \\
\hline 4. Estou suficientemente ocupado com tarefas (trabalho) significativas. & 3,775 & 1,030 & 0,468 \\
\hline $\begin{array}{l}\text { 5. Minhas tarefas são adequadamente desafiadoras para meu nível de conhecimento } \\
\text { e habilidades. }\end{array}$ & 3,806 & 1,081 & 0,495 \\
\hline 2. Meus supervisores estavam me esperando quando cheguei. & 3,538 & 1,264 & 0,511 \\
\hline $\begin{array}{l}\text { 24. Aqui, todos são tratados com igual respeito e dignidade, } \\
\text { independentemente de seu gênero. }\end{array}$ & 3,988 & 0,994 & 0,511 \\
\hline 22. A comunicação entre os que trabalham aqui é boa. & 3,453 & 0,950 & 0,531 \\
\hline 11. Os supervisores estão bem preparados para supervisionar. & 3,994 & 0,994 & 0,546 \\
\hline 21. Sinto-me bem-vindo na sala de funcionários/sala de refeições. & 3,270 & 1,153 & 0,552 \\
\hline 20. Aqui, sinto-me incluído na equipe de trabalho. & 3,308 & 1,143 & 0,555 \\
\hline 10. Tenho supervisores suficientes. & 3,888 & 1,135 & 0,555 \\
\hline 19. Como estudante, sou recebido de maneira positiva pela equipe. & 3,544 & 1,018 & 0,563 \\
\hline 17. Aqui, tenho a oportunidade de colocar meu conhecimento teórico em prática. & 4,025 & 0,987 & 0,583 \\
\hline $\begin{array}{l}\text { 15. Tenho a oportunidade de fundamentar minhas ações durante as sessões de } \\
\text { supervisão. }\end{array}$ & 3,856 & 1,018 & 0,590 \\
\hline 13. Recebo feedback útil dos meus supervisores. & 3,535 & 1,268 & 0,593 \\
\hline 9. Tenho um supervisor com quem sei que posso recorrer. & 3,944 & 1,074 & 0,615 \\
\hline 6. Sou incentivado a participar ativamente do trabalho. & 3,900 & 1,068 & 0,621 \\
\hline 26. Sinto que tenho influência sobre meu aprendizado nesse local de atuação. & 3,844 & 1,081 & 0,630 \\
\hline 3. Minhas tarefas (trabalho) são relevantes para os objetivos de aprendizagem. & 4,150 & 0,963 & 0,630 \\
\hline 12. Meus supervisores estão familiarizados com os objetivos de aprendizagem. & 4,194 & 0,925 & 0,642 \\
\hline $\begin{array}{l}\text { 16. Minhas habilidades de resolução de problemas estão se desenvolvendo bem } \\
\text { nesse local. }\end{array}$ & 3,963 & 0,948 & 0,644 \\
\hline
\end{tabular}

Fonte: dados da pesquisa. 
instrumento e a adaptação para a cultura da população-alvo disponibilizam instrumentos validados confiáveis para uso em diversos contextos e áreas.

A análise estatística possibilitou perceber que o instrumento traduzido e adaptado transculturalmente apresenta boa consistência interna, com correlação positiva entre as questões. Isso indica que quanto maior for o valor em cada item de forma individual, maior será o escore do resultado no instrumento como um todo. Esse é o padrão desejável com esse tipo de instrumento, pois, com ele, obtém-se uma escala de mensuração de satisfação do AE para ensino clínico dos estudantes, que varia do estudante com menor satisfação até aquele completamente satisfeito.

Considerando que a satisfação com o AE de ensino clínico tem relação com o desenvolvimento de competências clínicas, pode-se inferir que o instrumento se aplica para avaliar estudantes de diversos perfis acadêmicos. Isso significa que o instrumento possui validade em ampla faixa de perfis, de diferentes níveis de formação (períodos na graduação). Esse instrumento pode ser aplicado a estudantes ao longo da graduação, para acompanhar a satisfação do $A E$ de ensino clínico.

A satisfação com o desempenho das atividades acadêmicas pode ser considerada como engajamento, o qual é percebido no indivíduo pela energia despendida, pelo envolvimento e pela eficácia ao desempenhar determinada atividade, uma vez que seus valores são considerados seja no ambiente de trabalho ou nos estudos. Os estudantes engajados se sentem conectados às suas atividades e as percebem como um desafio positivo, pois variáveis como apoio social, autoeficácia, grau de satisfação pessoal e incentivo à autonomia auxiliam no desempenho e alcance dos objetivos acadêmicos ${ }^{19}$.

O ambiente de aprendizagem estimulante envolve os objetivos de aprendizagem, a parceria com os supervisores e oportunidades de interagir com os pacientes, além de permitir que os estudantes assumam responsabilidades. $\mathrm{O}$ bom relacionamento do supervisor/preceptor com o acadêmico e uma orientação eficaz são aspectos imprescindíveis no processo ensino-aprendizagem ${ }^{20}$.

A avaliação do AE nos Estados Unidos é frequentemente realizada nas escolas com objetivo de avaliar o clima educacional e a percepção dos estudantes e funcionários. A pesquisa apresenta por meio da percepção dos funcionários que o clima educacional tem influência nos resultados durante o dia de trabalho ${ }^{21}$.

No estudo realizado na Universiti Kebangsaan Malaysia Medical Centre, na Malásia, com estudantes do curso de Enfermagem, também relata que o bom relacionamento entre o supervisor e discente tem influência no processo ensino- aprendizagem, proporcionando uma melhor compreensão do conteúdo por parte do aluno ${ }^{22}$.

Com relação ao ensino clínico na saúde, a literatura indica a importância dos supervisores/preceptores, o preparo deles para a supervisão e a percepção do estudante sobre os supervisores. Há referência de que sejam profissionais capacitados e qualificados para supervisionar, visto que o estágio supervisionado/internato é uma oportunidade de o estudante expandir seus conhecimentos e colocá-los em prática. Os supervisores, além de inúmeras contribuições, precisam transmitir segurança no momento em que o estudante desenvolve as atividades na prática, enfatizando o que precisa ser melhorado ${ }^{9,23}$.

Em uma pesquisa, os estudantes relataram que o engajamento ativo no processo de aprendizado, o recebimento de supervisão clínica adequada e a valorização dos membros da equipe para ajudar a dar suporte ao atendimento ao paciente foram as expectativas em estágio supervisionado de obstetrícia e ginecologia ${ }^{24,25}$.

A supervisão realizada pelo professor no ensino clínico tem como objetivos preparar o estudante para sua prática profissional, proporcionar o engajamento com o ambiente em que está inserido e com a equipe de trabalho, desenvolver competências clínicas, planejar a sistematização da assistência e gestão de pessoas, contribuir na elaboração de ações educativas, entre outros ${ }^{26}$.

Quando o estudante está engajado com o AE e sente participante, isso contribui para o desempenho acadêmico e o crescimento pessoal e profissional dele ${ }^{27}$. Em pesquisa realizada na Universiti Kebangsaan Malaysia Medical Centre, constatou-se também que o $A E$ tem influência na aprendizagem do estudante, pois possibilita que ele adquira conhecimentos e habilidades que irão prepará-lo para sua prática profissional ${ }^{22}$.

No estudo realizado nos Estados Unidos, apreendeuse que o engajamento entre os funcionários de instituições de ensino proporciona um ambiente colaborativo, pois irão identificar objetivos em comum, e isso possibilitará desenvolver uma relação de confiança entre gestores e funcionários, contribuindo para que os colaboradores atinjam suas metas de trabalho com maior motivação ${ }^{21}$.

\section{CONCLUSÃO}

Foi possível alcançar os objetivos propostos pela pesquisa e responder a eles, para tradução, adaptação transcultural e validação da versão traduzida e adaptada para o português do Brasil com estudantes de Enfermagem e Medicina. Nesses dois cursos, a satisfação com o AE é essencial para o desenvolvimento do ensino clínico. 
A partir da validação, o instrumento pode ser aplicado a diversos cursos da área de saúde, possibilitando a outros pesquisadores e instituições de ensino o uso em seus contextos.

A TRI demonstrou-se eficiente em mensurar variáveis latentes que não podem ser verificadas diretamente. $\mathrm{O}$ instrumento validado apresentou perfil adequado para a avaliação de satisfação com o AE para ensino clínico em diversos perfis de estudantes. Nesse contexto, apreender a satisfação dos estudantes sobre o AE no ensino clínico implica adequar o $A E$ às expectativas do estudante de forma a melhor favorecer o aprendizado no ensino clínico.

$\mathrm{O}$ instrumento original possui quatro fatores, os quais foram mantidos na versão traduzida para a língua portuguesa do Brasil, mostrando-se confiável para ser utilizado em cursos de graduação da área de saúde para a Medida do Ambiente de Ensino Clínico na Graduação.

\section{CONTRIBUIÇÃO DOS AUTORES}

Milena da Costa e Ivete Palmira Sanson Zagonel participaram da concepção do estudo, da construção teórico-metodológica, da coleta e análise dos dados, da discussão e da revisão do artigo. Christian Boller participou da análise estatística dos dados, da discussão e da revisão do artigo.

\section{CONFLITO DE INTERESSES}

Declaramos não haver conflito de interesses.

\section{FINANCIAMENTO}

Declaramos não haver financiamento.

\section{REFERÊNCIAS}

1. Serrano-Gallardo P, Martínez-Marcos M, Espejo-Matorrales F, Arakawa T, Magnabosco GT, Pinto IC. Factors associated to clinical learning in nursing students in primary health care: an analytical cross-sectional study. Rev Latino-Am Enfermagem. 2016;24:e2803 [acesso em 03 set 2021]. Disponível em: https://www.scielo.br/pdf/rlae/v24/pt_0104-1169rlae-24-02803.pdf.

2. Troncon LEA. Ambiente educacional. Medicina (Ribeirão Preto). 2014;47(3):264-71.

3. Messas JT, Leonello VM, Fernandes MFP, Gonçalves GCC, Bucchi SM, Mira VL. O ambiente educacional do curso de graduação em enfermagem na perspectiva dos estudantes. Rev Esc Enferm USP. 2015;49(esp 2):10614 [acesso em 14 set 2021]. Disponível em: http://www.scielo.br/scielo. php?script=sci_arttext\&pid=S0080-62342015000800106\&lng=pt\&tlng=pt.

4. Hirsch CD, Barlem ELD, Barlem JGT, Silveira RS, Mendes DP. Fatores preditores e associados à satisfação dos estudantes de enfermagem. Acta Paul Enferm. 2015;28(6):566-72 [acesso em 03 set 2021]. Disponível em: http://www.scielo. br/scielo.php?script=sci_arttext\&pid=S0103-21002015000600566\&lng=pt\&t Ing=pt.

5. Damiance PRM, Tonete VLP, Daibem AML, Ferreira MLSM, Bastos JRM. Formação para o SUS: uma análise sobre as concepções e práticas pedagógicas em saúde coletiva. Trab Educ Saúde. 2016;14(3):699-721 [acesso em 17 set 2021]. Disponível em: https://www.scielo.br/pdf/tes/ v14n3/1678-1007-tes-14-03-0699.pdf.

6. Assis $A C L$, Assis JBL, Santos JMCT. Práticas educativas, memórias e oralidades. Rev do PEMO. 2021;3(2):1-12.
7. Fonseca MJ, Soares S, Gomes J, Marques A. O processo de supervisão em ensino clínico. Perspectiva dos estudantes e enfermeiros. Investig en Enfermería Imagen y Desarrollo. 2016;18(2):77-88.

8. Lima BPS, Vilela RQB. Características e desafios docentes na supervisão de estágio em fonoaudiologia. Rev CEFAC. 2014;16(6):1962-71.

9. Rigobello JL, Bernardes A, Moura AA, Zanetti ACB, Spiri WC, Gabriel CS. Supervised curricular internship and the development of management skills: a perception of graduates, undergraduates, and professors. Esc Anna Nery Rev Enferm. 2018;22(2):1-9.

10. Fouad S, El Araby $S$, Abed RAR, Hefny M, Fouad M. Using item response theory (IRT) to assess psychometric properties of undergraduate clinical education environment measure (UCEEM) among medical students at the Faculty of Medicine, Suez Canal University. Education in Medicine Journal. 2020;12(1):15-27. doi: 10.21315/eimj2020.12.1.3.

11. Acherman ND, Ribeiro AP, Lima LM, Cavalcanti ACD, Miranda TKQ, Oliveira GL. Mentoria entre pares: percepções de suporte social e ambiente educacional de estudantes de medicina. Rev Bras Educ Med. 2021;45(sup.1):e100. doi: 10.1590/1981-5271v45.supl.1-20210080.

12. Roberts $R$, Jennifer $C$, Strand P, Peter J. Medical students' views of clinical environments. Clin Teach. 2017;14:1-6. doi: 10.1111/tct.12691.

13. Strand $P$, Sjöborg $K$, Stalmeijer R, Wichmann-Hansen G, Jakobsson $\mathrm{U}$, Edgren G. Development and psychometric evaluation of the Undergraduate Clinical Education Environment Measure (UCEEM). Med Teach. 2013;35(12):1014-26.

14. Denise FP, Bernadette PH. Fundamentos da pesquisa em enfermagem. Porto Alegre: Artes Médicas; 1995.

15. Mamede FV, Prudêncio OS. Metodologias da pesquisa para a enfermagem e saúde: da teoria à prática. Porto Alegre: Moriá; 2015.

16. Beaton DT, Bombardier C, Guillemin FMB. Guidelines for the process of Cross Cultural adaptation of Self Report mesures. Spine (Phila Pa 1976). 2000;25(24):3186-91.

17. Samejima, F. Estimation of laten ability using a response pattern of graded scores. Psychometrika. 1969;34(4):100-14.

18. Tavakol M, Dennick R. Making sense of Cronbach's alpha. Int J Med Educ. 2011;2:53-5.

19. Silva JOM, Pereira Junior GA, Coelho ICMM, Picharski GL, Zagonel IPS Engajamento entre estudantes do ensino superior nas ciências da saúde (validação do questionário Ultrecht Work Engagement Scale (UWES-S) com estudantes do ensino superior nas ciências da saúde). Rev Bras Educ Med. 2018;42(2):15-25.

20. Hägg-Martinell $A$, Hult $H$, Henriksson $P$, Kiessling A. Medical students opportunities to participate and learn from activities at an internal medicine ward: an ethnographic study. BMJ Open. 2017;7(2):1-11.

21. Debnam KJ, Edwards K, Cornell D. Improving the school environment: school staff perceptions of school climate data and reporting practices. School of Nursing. J Community Psychol. 2021;49:1965-82. doi: 10.1002/ jcop. 22500 .

22. Karim J, Abdul Majid AHF, Mohd Rashdan NF, Awang Besar MN, Yaman MN. Nursing students' satisfaction towards clinical learning environment (CLE) in Universiti Kebangsaan Malaysia Medical Centre. Education in Medicine Journal. 2020;12(4):1-8. doi: 10.21315/eimj2020.12.4.1.

23. Maziero R. A contribuição do supervisor de estágio na formação dos estagiários. Acta Scientiae. 2010;14(1):63-75.

24. Mourão LF, Maria B, Teixeira V, Oliveira LB. Perspective of nursing students on curricular training. Rev Enferm UFPI. 2015;4(1):40-6.

25. Baecher-Lind LE, Chang K, Blanco MA. The learning environment in the obstetrics and gynecology clerkship: an exploratory study of students perceptions before and after the clerkship. Med Educ Online. 2015; 2981:104 [acesso em 16 set 2021]. Disponível em: https://www.tandfonline.com/ doi/pdf/10.3402/meo.v20.27273?needAccess=true.

26. Rocha EN, Mantovani MF, Silva ATM, Hereibi MJ, Viante WJM, Kwiatkosk DR. Percepção de competências clínicas por acadêmicos de Enfermagem. REME Rev Min Enferm. 2019, 23:e-1179. doi: 10.5935/1415-2762.20190027.

27. Rawas $\mathrm{H}$, Yasmeen N. Perception of nursing students about their educational environment in College of Nursing at King Saud Bin Abdulaziz University for Health Sciences, Saudi Arabia. Med Teach. 2019 Nov;41(11):1307-14. doi: 10.1080/0142159X.2019.1638502. 
ANEXO 1. Versão final do instrumento validado para o português do Brasil: Medida do Ambiente de Ensino Clínico na Graduação (MAECG).

\section{MAECG - "Medida do Ambiente de Ensino Clínico na Graduação"}

Strand P., Sjöborg K., Stalmeijer R., Wichmann-Hansen G., Jakobsson U., Edgren G. (2013). Desenvolvimento e avaliação psicométrica da medida do ambiente de educação clínica de graduação (UCEEM). Med Teach. 2013 Dec;35(12):1014-26.

Centro de ensino e aprendizagem, Faculdade de Medicina, Universidade de Lund, Suécia

Traduzido e adaptado: Costa. M.; Zagonel. I. P. S.

Faculdades Pequeno Príncipe - Programa de Pós-Graduação Stricto Sensu em Ensino nas Ciências da Saúde, Curitiba, Brasil.

Eu identifico meu gênero como:

( ) Feminino ( )Masculino ( )Outro ( )Prefiro não responder

Minha idade: ( )16-24 ( )25-34 ( )35 ou mais

Semestre: ( ) 3 ( ) 4 ( ) 5 ( ) 6 ( ) 7 ( ) 8 ( ) 9 ( ) 10 ( ) 11 ( ) 12

Meu local de atuação atual:

Considere as seguintes afirmações. Expresse seu ponto de vista marcando a caixa que você acredita que corresponde com maior precisão à sua percepção das condições em sua colocação clínica atual (ou local de atuação mais recente).
1. Recebi instruções iniciais úteis para este local de atuação.
( ) Discordo totalmente.
( ) Concordo um pouco.
( ) Neutro.
( ) Concordo em grande medida.
( ) Concordo plenamente.

2. Meus supervisores estavam me esperando quando cheguei.

( ) Discordo totalmente.

( ) Concordo um pouco.

( ) Neutro.

( ) Concordo em grande medida.

( ) Concordo plenamente.

3. Minhas tarefas (trabalho) são relevantes para os objetivos de aprendizagem.

( ) Discordo totalmente.

( ) Concordo um pouco.

( ) Neutro.

( ) Concordo em grande medida.

( ) Concordo plenamente.

4. Estou suficientemente ocupado com tarefas (trabalho) significativas.

( ) Discordo totalmente.

( ) Concordo um pouco.

( ) Neutro.

( ) Concordo em grande medida.

( ) Concordo plenamente.

5. Minhas tarefas são adequadamente desafiadoras para meu nível de conhecimento e habilidades.

( ) Discordo totalmente.

( ) Concordo um pouco.

( ) Neutro.

( ) Concordo em grande medida.

( ) Concordo plenamente.

6. Sou incentivado a participar ativamente do trabalho.

( ) Discordo totalmente.

( ) Concordo um pouco.

( ) Neutro.

( ) Concordo em grande medida.

( ) Concordo plenamente.

7. Eu tenho acesso adequado aos computadores.

( ) Discordo totalmente.

( ) Concordo um pouco.

( ) Neutro.

( ) Concordo em grande medida.

( ) Concordo plenamente. 
8. Há espaço físico suficiente para o número de estudantes da área de saúde alocados aqui.
( ) Discordo totalmente.
( ) Concordo um pouco.
( ) Neutro.
( ) Concordo em grande medida.
( ) Concordo plenamente.

9. Tenho um supervisor com quem sei que posso recorrer.

( ) Discordo totalmente.

( ) Concordo um pouco.

( ) Neutro.

( ) Concordo em grande medida.

( ) Concordo plenamente.

10. Tenho supervisores suficientes.
( ) Discordo totalmente.
( ) Concordo um pouco.
( ) Neutro.
( ) Concordo em grande medida.
( ) Concordo plenamente.
11. Os supervisores estão bem preparados para supervisionar.
( ) Discordo totalmente.
( ) Concordo um pouco.
( ) Neutro.
( ) Concordo em grande medida.
( ) Concordo plenamente.

12. Meus supervisores estão familiarizados com os objetivos de aprendizagem.
( ) Discordo totalmente.
( ) Concordo um pouco.
( ) Neutro.
( ) Concordo em grande medida.
( ) Concordo plenamente.

13. Recebo feedback útil dos meus supervisores.

( ) Discordo totalmente.

( ) Concordo um pouco.

( ) Neutro.

( ) Concordo em grande medida.

( ) Concordo plenamente.

14. Sinto-me à vontade em tirar dúvidas com meus supervisores de qualquer questão que deseje.

( ) Discordo totalmente.

( ) Concordo um pouco.

( ) Neutro.

( ) Concordo em grande medida.

( ) Concordo plenamente.

15. Tenho a oportunidade de fundamentar minhas ações durante as sessões de supervisão.
( ) Discordo totalmente.
( ) Concordo um pouco.
( ) Neutro.
( ) Concordo em grande medida.
( ) Concordo plenamente.

16. Minhas habilidades de resolução de problemas estão se desenvolvendo bem neste local.
( ) Discordo totalmente.
( ) Concordo um pouco.
( ) Neutro.
( ) Concordo em grande medida.
( ) Concordo plenamente.
17. Aqui, tenho a oportunidade de colocar meu conhecimento teórico em prática.
( ) Discordo totalmente.
( ) Concordo um pouco.
( ) Neutro.
( ) Concordo em grande medida.
( ) Concordo plenamente.


18. Aqui, tenho a oportunidade de aprender junto com outros estudantes da área de saúde.
( ) Discordo totalmente.
( ) Concordo um pouco.
( ) Neutro.
( ) Concordo em grande medida.
( ) Concordo plenamente.

19. Como estudante, sou recebido de maneira positiva pela equipe.

( ) Discordo totalmente.

( ) Concordo um pouco.

( ) Neutro.

( ) Concordo em grande medida.

( ) Concordo plenamente.

20. Aqui, sinto-me incluído na equipe de trabalho.

( ) Discordo totalmente.

( ) Concordo um pouco.

( ) Neutro.

( ) Concordo em grande medida.

( ) Concordo plenamente.

21. Sinto-me bem-vindo na sala de funcionários/sala de refeições.

( ) Discordo totalmente.

( ) Concordo um pouco.

( ) Neutro.

( ) Concordo em grande medida.

( ) Concordo plenamente.

22. A comunicação entre os que trabalham aqui é boa.

( ) Discordo totalmente.

( ) Concordo um pouco.

( ) Neutro.

( ) Concordo em grande medida.

( ) Concordo plenamente.

23. Aqui, todos são tratados com igual respeito e dignidade, independentemente de sua origem cultural.

( ) Discordo totalmente.

( ) Concordo um pouco.

( ) Neutro.

( ) Concordo em grande medida.

( ) Concordo plenamente.

24. Aqui, todos são tratados com igual respeito e dignidade, independentemente de seu gênero.

( ) Discordo totalmente.

( ) Concordo um pouco.

( ) Neutro.

( ) Concordo em grande medida.

( ) Concordo plenamente.

25. Aqui, todos são tratados com igual respeito e dignidade, independentemente de sua profissão.
( ) Discordo totalmente.
( ) Concordo um pouco.
( ) Neutro.
( ) Concordo em grande medida.
( ) Concordo plenamente.

26. Sinto que tenho influência sobre meu aprendizado nesse local de atuação.

( ) Discordo totalmente.

( ) Concordo um pouco.

( ) Neutro.

( ) Concordo em grande medida.

( ) Concordo plenamente.

Por favor, comente sobre outros aspectos de importância para o ambiente educacional nesta local de atuação e dê sugestões de como ele pode ser melhorado:

Legenda: Entende-se por cursos da área de saúde: Medicina, Enfermagem, Farmácia, Nutrição, Fisioterapia, Odontologia, Psicologia, Terapia Ocupacional, Gerontologia, Estética, Biomedicina, Educação Física, Saúde Coletiva. Responder de acordo com o seu curso. 